\title{
Electron Pairing and Chemical Bonds. Electron Fluctuation and Pair Localization in ELF Domains
}

\author{
ROBERT PONEC, ${ }^{1}$ JOAQUIN CHAVES ${ }^{2}$ \\ ${ }^{1}$ Institute of Chemical Process Fundamentals, Czech Academy of Sciences, Prague 6, \\ Suchdol 2, 165 02, Czech Republic \\ ${ }^{2}$ Institute of Computational Chemistry, University of Girona, Campus Montilivi, 17071, \\ Girona, Spain \\ Received 10 February 2005; accepted 11 April 2005 \\ DOI 10.1002/jcc.20257 \\ Published online in Wiley InterScience (www.interscience.wiley.com).
}

\begin{abstract}
This article reports the numerical comparison of the quantities characterizing the extent of electron fluctuation and pair localization in the domains determined by the direct minimization of electron fluctuation with the domains resulting from the partitioning of the molecules based on the topological analysis of the so-called electron localization function (ELF). Such a comparison demonstrates that the ELF partitioning can be regarded as a feasible alternative to computationally much more demanding direct optimization of minimum fluctuation domains. This opened the possibility of the systematic scrutiny of the electron pair model of the chemical bond, and as it was demonstrated, the previous pessimistic claims about the applicability of this model are not completely justified.
\end{abstract}

(C) 2005 Wiley Periodicals, Inc. J Comput Chem 26: 1205-1213, 2005

Key words: electron pairing; chemical bonds; ELF domains

\section{Introduction}

Since its introduction by Lewis, ${ }^{1}$ the idea of a chemical bond as a shared electron pair has played the pivotal role in the conceptual development of the modern chemistry. Because of immense debts that chemistry owes to the classical picture of localized chemical bonds joining the bonded atoms, the Lewis model survived the advent of quantum mechanics with its concept of delocalized wave function spread over the whole molecule, and a lot of effort was devoted to the reconciliation of these two alternative pictures of bonding. ${ }^{2-12}$ In addition to earlier studies in which the apparent discrepancy between localized classical and delocalized quantum mechanical description of bonding has been addressed using the idea of localized molecular orbitals, ${ }^{13-21}$ there is another group of approaches based in a sense on the original idea of Daudel et al. $^{22-24}$ known as the loge theory. According to this theory, there should be some "best" partitioning of the molecular space into a number of disjunct domains, the so-called loges, which thus represent the most probable partitioning (in quantum mechanical sense) of the molecules into localized groups of electrons. The boundaries of these individual domains are to be determined from the condition of the minimization of the so-called missing information function. ${ }^{25}$ This conceptually very attractive approach is, however, computationally very demanding, and especially in the time it was proposed, its application was beyond the reach of available computational means. A certain simplification of this approach was offered when Daudel et al. ${ }^{5}$ reported that the partitioning based on the minimization of the missing information function is closely paralleled by the partitioning based on the minimization of electron fluctuation in individual domains. This approach was thoroughly studied by Bader et al., ${ }^{26,27}$ who attempted to determine whether or to what extent the "best" partitioning into the localized domains reflects the classical concepts of core, bond, and/or lone electron pairs. Nevertheless, because of nonavailability of sufficiently powerful algorithm for the explicit optimization of the form of the domains corresponding to minimum electron fluctuation, he was able to perform the analysis only for several very simple molecules in which the form of the domains could be reasonably deduced on the basis of symmetry arguments. The conclusions of this study were nevertheless quite discouraging, because it was found that the satisfactory partitioning of the molecule into domains with high localization of electron pairs is possible only in the very simplest cases, while in some others, even slightly more complex molecules, the localized pair model ceases to afford a suitable description. ${ }^{27}$

Correspondence to: R. Ponec; e-mail: rponec@icpf.cas.cz Contract/grant sponsor: Grant Agency of the Czech Academy of Sciences; contract/grant number: IAA4072403 
Our aim in this study is to reconsider the conclusions of the study ${ }^{27}$ and to demonstrate that the above claims are apparently too pessimistic. Such reconsideration is motivated by the results of several more recent studies, ${ }^{11,28,29}$ in which the possibility of the reduction of quantum mechanical wave function to indices straightforwardly reflecting the classical structural formula was convincingly demonstrated.

In addition to this primary goal, another aim of this study is to demonstrate that so-far unfeasible partitioning of the molecule into the domains of minimum electron fluctuation can reasonably be approximated using the so-called electron localization function (ELF). ${ }^{12,30}$ This function, namely, allows the unique partitioning of the molecule into disjunct domains, which, as it will be shown, are surprisingly similar to the domains of minimum electron fluctuation determined, where possible, by the direct optimization. ${ }^{27}$ This result is very important, because the ELF partitioning is easily available for practically any molecule. The basic idea of the loge theory can thus be subject to more extensive and more systematic scrutiny than was possible in the study. ${ }^{27}$ As it will be shown, the original claims about the impossibility of the representation of the molecule as composed of chemical bonds are not completely justified, and the classical Lewis model of chemical bond need not be dismissed.

\section{Theoretical}

As demonstrated by Bader et al., ${ }^{26,27}$ the extent of electron localization in a region $\Omega$ can quantitatively be characterized by the value electron fluctuation in the same region. This fluctuation is defined by eq. (1)

$$
\Lambda(\Omega)=\overline{N(\Omega)}+F(\Omega, \Omega)
$$

in which $\overline{N(\Omega)}$ denotes the mean number of electrons in the domain $\Omega$ given by the integral of the first order density $\rho\left(r_{1}\right)$ over that domain [eq. (2)],

$$
\overline{N(\Omega)}=\int_{\Omega} \rho\left(r_{1}\right) d r_{1}
$$

and the quantity $F(\Omega, \Omega)$, is defined in terms of pair density $\rho\left(r_{1}\right.$, $r_{2}$ ) by eq. (3),

$$
F(\Omega, \Omega)=2 \int_{\Omega} d r_{1} \int_{\Omega} \rho\left(r_{1}, r_{2}\right) d r_{2}-\overline{N(\Omega)^{2}}
$$

Using this quantity, the extent of pair localization can be characterized, for example, by the quantity $g(\Omega)$ [eq (4)]

$$
g(\Omega)=-\frac{F(\Omega, \Omega)}{\overline{N(\Omega)}}
$$

whose values vary between $0-1$. The closer the value of the index is to 1 , the better is the electron pair localized in the domain $\Omega$.
These equations are completely general, and remain valid at any level of the theory irrespective of the form of the domain $\Omega$. In the particular case that $\Omega$ involves the whole space $\left(\left(\Omega_{T}\right)\right.$, the electron fluctuation identically equals to zero.

Within the framework of SCF approximation, which is of our concern here, the pair density can explicitly be calculated using the first-order density matrix and the general formula (1) reduces to eq. (5)

$$
\Lambda(\Omega)=\overline{N(\Omega)}-2 \sum_{i}^{\text {occ }} \sum_{j}^{\text {occ }}\langle i \mid j\rangle_{\Omega}^{2}
$$

where the symbol $\langle i \mid j\rangle_{\Omega}$ denotes the overlap integral of (occupied) molecular orbitals $\varphi_{i}$ and $\varphi_{j}$ over the domain $\Omega$ [eq. (6)],

$$
\langle i \mid j\rangle_{\Omega}=\int_{\Omega} \varphi_{i}(r) \varphi_{j}(r) d r
$$

In this study the suitability of ELF domains to represent the partitioning of molecule into fragments that can be regarded as reasonable approximations to the domains of minimum electron fluctuation will be primarily demonstrated on the basis of comparison of actual numerical values of domain populations, fluctuations, and localization indices $g(\Omega)$. In addition to such a straightforward comparison there is, however, yet another possibility that allows in a sense to evaluate the "quality" of both these alternative partitionings. This possibility is based on the following simple idea: let us imagine first that the molecular space of a given molecule is partitioned into a set of nonoverlapping domains $\Omega_{\alpha}$ is such a way that their union restores again the whole space $\Omega_{T}$. The number and the actual form of individual domains is in this moment irrelevant. In this case, the original eq. (5) can be rewritten in the form (7)

$$
0=\Lambda\left(\Omega_{T}\right)=\sum_{\alpha} \Lambda\left(\Omega_{\alpha}\right)+2 \sum_{\alpha<\beta} F\left(\Omega_{\alpha}, \Omega_{\beta}\right),
$$

in which the quantity $\Lambda\left(\Omega_{\alpha}\right)$ denotes the fluctuation of electrons in the domain $\Omega_{\alpha}$. If we now take into account that the quantities $F\left(\Omega_{\alpha}, \Omega_{\beta}\right)$ are always negative [eq. (8)],

$$
F\left(\Omega_{\alpha}, \Omega_{\beta}\right)=-2 \sum_{i}^{\text {occ occ }} \sum_{j}\langle i \mid j\rangle_{\Omega_{\alpha}}\langle j \mid i\rangle_{\Omega_{\beta}}
$$

eq. (7) implies that the electron fluctuation in a joint region is always smaller than the sum of fluctuations in the individual domains. This result is very important because it implies that electrons can never be completely localized in individual domains, or, which is equivalent, the electrons is individual domains can never be considered as completely independent. In this sense the scepticism of Bader et al. ${ }^{26,27}$ concerning the possibility of complete localizability of electrons is indeed justified. Despite this, we would nevertheless like to show that the classical model of molecule as composed of localized chemical bonds need not be com- 
Table 1. Comparison of Domain Populations, Fluctuations, and Pair Localization in ELF Domains with the Same Data from the Study. ${ }^{27}$

\begin{tabular}{|c|c|c|c|c|c|}
\hline Molecule & Domain & Method & $\overline{N(\Omega)}$ & $\Lambda(\Omega)$ & $g(\Omega)$ \\
\hline \multirow[t]{4}{*}{$\mathrm{LiH}$} & \multirow[t]{2}{*}{$\mathrm{C}(\mathrm{Li})$} & ELF & 2.01 & 0.06 & 0.97 \\
\hline & & Study $^{27}$ & 1.999 & 0.091 & 0.96 \\
\hline & \multirow[t]{2}{*}{$\mathrm{V}(\mathrm{LiH})$} & ELF & 1.98 & 0.07 & 0.96 \\
\hline & & Study $^{27}$ & 2.001 & 0.091 & 0.96 \\
\hline \multirow[t]{4}{*}{$\mathrm{BeH}_{2}$} & \multirow[t]{2}{*}{$\mathrm{C}(\mathrm{Be})$} & ELF & 2.03 & 0.13 & 0.94 \\
\hline & & Study $^{27}$ & 2.012 & 0.137 & 0.93 \\
\hline & \multirow[t]{2}{*}{$\mathrm{V}(\mathrm{BeH})$} & ELF & 1.98 & 0.14 & 0.93 \\
\hline & & Study $^{27}$ & 1.994 & 0.144 & 0.93 \\
\hline \multirow[t]{4}{*}{$\mathrm{BH}_{4}^{(-)}$} & \multirow[t]{2}{*}{$\mathrm{C}(\mathrm{B})$} & ELF & 2.07 & 0.19 & 0.91 \\
\hline & & Study $^{27}$ & 2.026 & 0.212 & 0.90 \\
\hline & \multirow[t]{2}{*}{$\mathrm{V}(\mathrm{BH})$} & ELF & 1.98 & 0.45 & 0.77 \\
\hline & & Study $^{27}$ & 1.994 & 0.472 & 0.76 \\
\hline
\end{tabular}

pletely dismissed. For this purpose let us return to eq. (7). This equation implies, namely, that to keep the fluctuation over the whole space zero, the sum of fluctuations over individual domains must be exactly compensated by the second term involving the quantities $F\left(\Omega_{\alpha}, \Omega_{\beta}\right)$, which characterize the extent of interactions between the electrons in different individual domains. The existence of this exact cancellation thus opens the possibility to characterize the extent of localization in individual domains and, in a sense, the "quality" of a given partitioning, on the basis of the value of the sum of the fluctuations over individual subdomains. The smaller is, namely, this sum, the smaller compensation in terms of $F\left(\Omega_{\alpha}, \Omega_{\beta}\right)$ quantities is required and, consequently, the better the localization of the electrons in individual domains. Based on this criterion, the "quality" of the partitioning of the molecule into ELF and directly optimized domains can straightforwardly be evaluated.

Although, the ELF partitioning of molecules is generally able to provide the picture of the bonding, which is very reminiscent of classical Lewis model of localized chemical bonds, it is nevertheless fair to say that there are also some examples where the above parallel is less convincing. The reasons for these discrepancies are not at this moment completely clear, but we believe that rather than indicating the failure of the parallel between chemical bonds and ELF domains, these exceptions can apparently be regarded as the demonstrations of the insufficiency of the actual definition of electron localization function to determine the boundaries of individual domains realistically enough in all molecules. In this connection it is interesting to mention the recent study by Savin et al. ${ }^{31}$ in which a similar discrepancy between the ELF domains and the domains of maximum probability was reported.

\section{Calculations}

The suitability of ELF domains to represent the partitioning of the molecule into localized electron pairs reminiscent of classical Lewis model of chemical bond was tested on a series of simple molecules involving the following model systems: (a) molecules for which the satisfactory localization of electron pairs was pos- sible ${ }^{27}\left(\mathrm{LiH}, \mathrm{BeH}_{2}, \mathrm{BH}_{4}^{(-)}\right)$; (b) bigger molecules for which the optimization of the form of domains of minimum electron fluctuation would be directly unfeasible $\left(\mathrm{C}_{2} \mathrm{H}_{6}, \mathrm{C}_{2} \mathrm{H}_{4}, \mathrm{C}_{2} \mathrm{H}_{2}\right)$; (c) molecules for which the localization of electron pairs was claimed to be unacceptably small ${ }^{27}\left(\mathrm{H}_{2} \mathrm{O}, \mathrm{N}_{2}\right)$.

The analysis of bonding in these systems required to perform two types of calculations. First, it was necessary to generate the reliable wave functions for all of the studied systems. These calculations were performed using Gaussian 98 series of programs, ${ }^{32}$ at the HF/6-31G** level of the theory. This level of theory was chosen to correspond to the computational standards of the study ${ }^{27}$ with which our results will be directly compared. All the molecules were considered in completely optimized molecular geometries based on these preliminary calculations that the subsequent analysis of the fluctuations in ELF and AIM domains was performed using the TopMod program. ${ }^{33}$ The results of the calculations are summarized in Tables $1-5$.

\section{Results and Discussion}

After having introduced the basic methodological background of the presented approach, let us discuss now the results of our analyses for individual systems. As the first example let us consider the molecules of $\mathrm{LiH}, \mathrm{BeH}_{2}$, and $\mathrm{BH}_{4}^{(-)}$, for which the satisfactory localization of electron pairs was found in the study. ${ }^{27}$ The results of such a comparison are summarized in Table 1 . Before discussing the data from this table it is, however, useful to say a few words of explanation concerning the procedure used in the study ${ }^{27}$ for the determination of the form of the domains of minimum fluctuation. Because of nonavailability of the general computational procedure for the optimization of the form of these domains, the partitioning was performed using the following semiempirical procedure: First, it was assumed that the domain corresponding to core-electron pair is spherical, and the radius of this sphere was obtained from the condition of the minimum electron fluctuation. The bond domains were then determined by the partitioning of the remaining valence space on the basis of appropriate symmetry considerations. Such a procedure is cer- 
tainly quite satisfactory from the practical point of view, but strictly speaking, such an approach is nonetheless a bit arbitrary. Moreover, it is clear that such a procedure can be applied only to very simple systems with sufficient symmetry. On the other hand, the ELF partitioning is free of any such limitations and can be applied to practically any molecule.

\section{$\mathrm{LiH}$}

This molecule represents an example of the systems for which the partitioning into localized electron pairs is indeed extremely trivial. The reason is that after separating the domain corresponding to core electrons on $\mathrm{Li}$, there is just one additional pair that has to be associated with the $\mathrm{LiH}$ bond and the domain corresponding to this electron pair then necessarily must occupy the reminder of the space. The populations of electrons in these intuitively determined domains are equal to $1.999(\mathrm{Li})$ and $2.001(\mathrm{LiH})$, and the corresponding fluctuations are equal to 0.091 for each of these domains. ${ }^{27}$ Let us confront now this result with the picture of bonding based on the partitioning into ELF domains. Such partitioning yields in this case also two domains (Table 1). One of them corresponds again to core $1 \mathrm{~s}^{2}$ electron pair on $\mathrm{Li}$, and the other can be classified as disynaptic domain ${ }^{34}$ corresponding to electron pair of $\mathrm{LiH}$ bond. The populations of both domains (2.01 for $\mathrm{C}(\mathrm{Li})$, 1.98 for $\mathrm{V}(\mathrm{LiH})$ ) are in this case slightly different than in the previous case, but this difference, which may apparently be attributed to the slight arbitrariness assuming the strictly spherical form of the core domain in ref. 27, is not dramatic. (The deviations from the strict sphericity of the core domain of $\mathrm{Li}$ in $\mathrm{LiH}$ was recently reported in the study. ${ }^{31}$ ) The same similarity is also evident from the comparison of the fluctuations in the individual domains $(0.06$ and 0.07 , respectively). Also, in this case, there are slight differences between the fluctuations in both types of domains, but the difference is again only of marginal importance. Very similar also are the values of the parameter $g(\Omega)$ from which the high degree of pair localization in both ELF and directly optimized domains is again straightforwardly evident. We can thus see that the partitioning into ELF domains is indeed in all aspects very reminiscent of the partitioning based on the direct optimization of the domains of minimum electron fluctuation. ${ }^{27}$ This is also the case of the remaining simple systems $\mathrm{BeH}_{2}$ and $\mathrm{BH}_{4}^{(-)}$.

\section{$\mathrm{BeH}_{2}$}

Another example of the molecule for which the satisfactory partitioning into the domains corresponding to individual localized electron pairs was found in the study ${ }^{27}$ is the molecule of $\mathrm{BeH}_{2}$. The partitioning of this six-electron molecule was again very straightforward. One of the pairs was assumed to correspond to $1 \mathrm{~s}^{2}$ core electron pair on the Be atom, and the other has to be necessarily associated with the $\mathrm{BeH}$ bonds. The populations of these domains, together with the corresponding fluctuations and localization parameters $g(\Omega)$, are summarized again in Table 1. The same table also contains the corresponding data for the alternative ELF partitioning. As it is possible to see, the results of both alternative partitionings are again very similar in all aspects.
Table 2. Comparison of the ELF and Directly Determined Domains of Minimum Electron Fluctuation from the Point of View of Independence of Electron Pairs in Individual Domains.

\begin{tabular}{lll}
\hline Molecule & Partitioning & $\sum \Lambda(\Omega)$ \\
\hline $\mathrm{LiH}$ & ELF & 0.13 \\
& Study $^{27}$ & 0.182 \\
$\mathrm{BeH}_{2}$ & ELF $^{27}$ & 0.41 \\
& Study $^{27}$ & 0.425 \\
$\mathrm{BH}_{4}^{(-)}$ & ELF & 1.99 \\
& Study $^{27}$ & 2.101 \\
\hline
\end{tabular}

$$
\mathrm{BH}_{4}^{(-)}
$$

The largest molecule for which the electron pair model of chemical bond was found plausible ${ }^{27}$ is the molecule of $\mathrm{BH}_{4}^{(-)}$. The partitioning into the individual pairs was in the study ${ }^{27}$ again obtained using the above-reported semiexact procedure. Such a partitioning yielded the following populations of the individual domains: 2.026 for the core pair on B and 1.994 for the $\mathrm{BH}$ bond, and the corresponding fluctuations are 0.212 and 0.472 , respectively (Table 1). Qualitatively very similar is also the partitioning into ELF domains; the only slight difference is in the values of domain populations and fluctuations (Table 1). On the other hand, the values of localization parameters $g(\Omega)$ for both types of domains are practically the same. We can thus again see that the partitioning of the molecule into ELF domains can indeed be regarded as a good approximation to the partitioning into domains of minimum electron fluctuation.

After having demonstrated the close parallel between the partitioning into ELF domains and the domains of minimum electron fluctuation in these molecules, let us look at the similarity of both partitionings from another point of view. For this purpose let us concentrate on the comparison of the sum of the fluctuations over the individual domains. Such a comparison demonstrates (Table 2) that although these values are generally very similar, one can nevertheless notice a clear trend that the sum of the fluctuations over ELF domains is even slightly lower than the sum of fluctuations over the domains of minimum fluctuation. ${ }^{27}$ This result is very important, because it suggests that due to slight arbitrariness, the procedure used for the determination of the form of the domains in ref. 27, does not apparently yield the really "best" domains and the ELF partitioning seems to be, in fact, slightly superior. This implies, however, that ELF partitioning can be regarded not only as a mere approximation, but really as a feasible approach to the partitioning of the molecule anticipated in the loge theory. This is especially interesting for the more complex molecules where the original semiempirical procedure of the study ${ }^{27}$ is not applicable. In the following part we are going to present the comparison of various domain characteristics for several such more complex molecules.

Before reporting the results of these calculations it is, however, useful to discuss in more details the general trends observed in the values of localization parameter $g(\Omega)$ in Table 1 . As it is possible to see, this parameter is generally largest for the electron pairs associated with the core electrons of the heavy atom, and in all 
Table 3. Domain Populations, Fluctuations, and Pair Localization Parameters for the ELF Domains of Several Simple Molecules for Which the Direct Procedure Used in the Study ${ }^{27}$ Is Not Feasible.

\begin{tabular}{lllll}
\hline Molecule & Domain & $\overline{N(\Omega)}$ & $\Lambda(\Omega)$ & $g(\Omega)$ \\
\hline $\mathrm{C}_{2} \mathrm{H}_{6}$ & $\mathrm{C}(\mathrm{C})$ & 2.09 & 0.25 & 0.88 \\
& $\mathrm{~V}(\mathrm{CH})$ & 2.00 & 0.62 & 0.69 \\
& $\mathrm{~V}(\mathrm{CC})$ & 1.83 & 0.94 & 0.49 \\
$\mathrm{C}_{2} \mathrm{H}_{4}$ & $\mathrm{C}(\mathrm{C})$ & 2.09 & 0.22 & 0.88 \\
& $\mathrm{~V}(\mathrm{CH})$ & 2.08 & 0.61 & 0.71 \\
& $\mathrm{~V}(\mathrm{CC})$ & 1.76 & 0.95 & 0.46 \\
$\mathrm{C}_{2} \mathrm{H}_{2}$ & $\mathrm{C}(\mathrm{C})$ & 2.09 & 0.24 & 0.88 \\
& $\mathrm{~V}(\mathrm{CH})$ & 2.28 & 0.66 & 0.71 \\
& $\mathrm{~V}(\mathrm{CC})$ & 5.27 & 1.32 & 0.75 \\
& & & &
\end{tabular}

case the actual values exceed 0.90 . This result is not at all surprising-it only straightforwardly reflects the fact that core electrons are indeed well separated from the valence electrons. On the other hand, the localization valence electrons in bond domains is much more molecule sensitive, and the localization parameters drop from relatively high value for bond pair in $\mathrm{LiH}(0.96)$ to the considerably lower value of 0.77 for the $\mathrm{BH}$ bond domain in $\mathrm{BH}_{4}^{(-)}$. Similarly, the low localization parameter $g(\Omega)$ was also found in the case of the bond domain in $\mathrm{CH} 4{ }^{27}$ and the actual value 0.69 , was even claimed to represent the borderline case for which the concept of the localized electron pair starts to lose its meaning.

Our aim in this study is to show that, despite the observed decrease, the above interpretation of the pair localization parameter $g(\Omega)$ is apparently oversimplified and does not necessarily imply the failure of the concept of electron pair bond. To corroborate this claim, let us discuss as a first example the molecule of ethane.

\section{$\mathrm{C}_{2} \mathrm{H}_{6}$}

This molecule represents a simple example of the system for which the procedure used for the optimization of minimum fluctuation domains would not be applicable. Nevertheless, the ELF partitioning into localized electron pairs reminiscent of the classical chemical bonds of the Lewis model is straightforwardly possible, and as it will be shown, the quantities characterizing the population, fluctuation, and pair localization of ELF domains are, at least for core and $\mathrm{CH}$ bond electron pairs where the comparison is possible, very similar to what was reported for the closely related molecule of $\mathrm{CH}_{4}$ in the study. ${ }^{27}$ The high probability of localizing the electron pairs in ELF domains was also clearly demonstrated in the recent study by Chamorro et al. ${ }^{35}$

The results of our analysis for this molecule are summarized in Table 3. The ELF partitioning of this 18-electron molecule yields indeed nine roughly two-electron domains. Two of them, populated by 2.09 electrons, correspond to core $1 \mathrm{~s}^{2}$ electron pairs on individual carbons. The calculated fluctuation in these domains (0.25) is very close to what was found in ref. 27 for carbon core of methane (0.239), and practically the same is also the value of the localization parameter $g(\Omega)(0.88)$. The same close similarity is also observed for the domains $\mathrm{V}(\mathrm{CH})$ associated with electron pairs of $\mathrm{CH}$ bonds. Each of these domains is populated by roughly 2.00 electrons (compared to 1.999 in the study ${ }^{27}$ ) and very similar also are the corresponding fluctuations ( 0.62 vs. 0.622 in ref. 27$)$ and pair localization parameters. The only remaining domain completing the set of nine electron pairs in this molecule thus corresponds to the CC bond. The population of this domain (1.83) is a bit lower than in the previous case of $\mathrm{CH}$ bonds, but still the parallel with the electron pair bond is reasonably plausible. The slight decrease in the population of this domain is then reflected by the accompanying increase of the domain fluctuation (0.94), but unfortunately, there are no reference data with which these values could be compared. The same is also true of the pair localization parameter $g(\Omega)$, whose value drops to 0.49 . Although this parameter is even considerably lower than the limiting value 0.69 considered in the study, ${ }^{27}$ we believe that the straightforward interpretation in a sense of the complete failure of the concept of electron pair bond is not completely justified.

To corroborate this claim let us look at the problem of pair localization from another point of view. For this purpose let us raise the question of what maximum localizability of electron pairs can be expected, if the complete localization is impossible? To answer such a question it is important to realize that instead of insisting on "sufficiently" high pair localization in each individual domain, it is more important to concentrate on a global description and to ask what is the possibility of the pair localization for the molecule as a whole.

A quantity allowing such a global characterization is the sum of the fluctuations over all the domains. The lower this sum, the smaller the interactions between the electrons in individual domains, characterized by the values of the interdomain correlation terms $F\left(\Omega_{\alpha}, \Omega_{\beta}\right)$ and the more "independent" these domains are. The complete pair localizability would require these terms to vanish so that the impossibility of complete pair localization also implies that interactions between individual domains can never be ignored. The importance of these interaction terms was clearly demonstrated in the study, ${ }^{28}$ in which we have shown that in the case of partitioning of the molecule into atomic AIM domains, the existence of the covalent bond between a given pair of atoms is crucially connected just with the existence of nonvanishing interdomain interactions between the corresponding atoms. In the case of directly bonded atoms, these interdomain interaction terms were even shown to coincide with the classical bond multiplicities, while the interactions between classically nonbonded atoms are often negligible. Similar interplay between negligible and nonnegligible interdomain interaction terms can also evidently be expected in the case of the partitioning to ELF domains, and in the following part we attempt to elucidate the manifestation of bonding interactions in the case of partitioning into such domains. The chemical bonds (shared electron pairs) are in this case described by intradomain correlation terms $F\left(\Omega_{\alpha}, \Omega_{\alpha}\right)$, and the physical meaning of interdomain interactions is not as straightforward as in the case of AIM partitioning. This, however, does not mean that the clear interpretation of these terms cannot be given and, especially that the applicability of electron pair model of chemical bond should anyhow rely on the absence of such interactions. To elucidate this problem let us imagine the model situation that the valence electrons could be localized to electron pair bonds to the same high extent as it is usual for the core pairs. If this were 
Table 4. Calculated Values of Interdomain Interaction Terms $F\left(\Omega_{\alpha}, \Omega_{\beta}\right)$ for the ELF Partitioning of the $\mathrm{C}_{2} \mathrm{H}_{6}, \mathrm{C}_{2} \mathrm{H}_{4}$, and $\mathrm{C}_{2} \mathrm{H}_{2}$ Molecules.

\begin{tabular}{lllll}
\hline & \multicolumn{4}{c}{$\Omega_{\mathrm{B}}$} \\
\cline { 2 - 5 }$\Omega_{\mathrm{A}}$ & $\mathrm{C}\left(\mathrm{C}^{\prime}\right)$ & $\mathrm{V}(\mathrm{CC})$ & $\mathrm{V}(\mathrm{CH})_{\text {adjacent }}$ & $\mathrm{V}(\mathrm{CH})_{\text {nonadjacent }}$ \\
\hline Molecule: $\mathrm{C}_{2} \mathrm{H}_{6}$ & & & & \\
$\quad \mathrm{C}(\mathrm{C})$ & -0.001 & -0.051 & -0.062 & -0.003 \\
$\mathrm{~V}(\mathrm{CH})$ & & -0.140 & -0.183 & -0.015 \\
Molecule: $\mathrm{C}_{2} \mathrm{H}_{4}$ & & & & -0.006 \\
$\mathrm{C}(\mathrm{C})$ & -0.002 & -0.048 & -0.067 & -0.029 \\
$\mathrm{~V}(\mathrm{CH})$ & & -0.149 & -0.184 & \\
$\mathrm{~V}(\mathrm{CC})$ & & -0.147 & & -0.012 \\
Molecule: $\mathrm{C}_{2} \mathrm{H}_{2}$ & & & & -0.051 \\
C(C) & -0.003 & -0.147 & -0.082 & - \\
V(CH) & & -0.513 & - & \\
\hline
\end{tabular}

possible, then such a set of highly localized electron pairs could not be considered as a molecule-there would be no chemical bonding possible for the same reasons that prevent the existence of chemical bond between core electrons. This result is very important, because it clearly implies that the original criterion conditioning the applicability of electron pair model of chemical bond by "sufficiently" high pair localization in each of the individual domains ${ }^{27}$ is not apparently reasonable as a test of justification of the electron pair model of the chemical bond. In other words, the fact that the pair localization in some domain is found "unacceptably" small cannot be regarded as a failure of the electron pair model of the chemical bond.

To demonstrate the role of the interdomain interaction terms in chemical bonding for the case of ELF partitioning, let us discuss the actual values of these quantities for the ethane molecule. The corresponding data are summarized in Table 4. As it is possible to see, a lot of the interdomain interactions is indeed quite small. This is, for example, the case of interactions between core domains of different $\mathrm{C}$ atoms and, also between the core domain of one of the carbons and the domains associated with electron pairs of any $\mathrm{CC}$ and $\mathrm{CH}$ bonds. In addition to this anticipated result, which again only reflects the well-known fact that the involvement of core electrons in bonding is generally negligible, another example of weakly interacting pairs are the electron pairs of nonadjacent $\mathrm{CH}$ bonds, that is, the bonds localized on different carbon atoms. This result is very interesting because it suggests that nonadjacent bonds can, even in the case of ELF partitioning, be considered as more or less independent. To require the same or similar independence also for the adjacent bonds localized on the same atom would be, however, too restrictive because it would imply the absence of all interactions in the molecule, and the situation would thus correspond to what was rejected in the above model example of noninteracting core electron pairs. The existence of at least some nonvanishing interdomain interactions is thus, in this case, crucially essential for the presence of bonding interactions in the molecule. A straightforward consequence of the existence of nonnegligible interdomain interaction terms is then the increase of the fluctuation in certain, usually bond domains, which is reflected in the observed decrease of the localization parameter $g(\Omega)$. The decrease of this parameter for certain domains should not thus be regarded as a factor that destroys the electron pair model of chemical bond, but rather as a fundamental condition, without which the phenomenon of chemical bonding would be impossible.

In this connection it is, of course, necessary to address the apparent inconsistency between the above claims and the fact that relatively very high pair localization was found in molecules like $\mathrm{LiH}$ and $\mathrm{BeH}_{2}$. For this purpose it is useful to discuss the nature of bonding in these molecules in more details. The simplest situation is in the case of $\mathrm{LiH}$. In this case there are, namely, only two domains. One of them corresponds to core electron pair on $\mathrm{Li}$ and the other is the $\mathrm{LiH}$ "bond" domain. In this trivial case eq. (7) implies the existence of only one interdomain interaction term corresponding to interactions between core and bond domains. Such terms are, however, generally very small (actual value is $-0.065)$. As a consequence, the sum of the fluctuations in both individual domains must also be small so that the pair localization in both domains is necessarily high. Such a situation is, however, possible only because of the high polarity of the $\mathrm{LiH}$ bond. The electron distribution in this bond resembles the limit of the completely ionic bond, and in fact, the ELF domain of the LiH bond is very similar to the AIM atomic domain of $\mathrm{H}$ in $\mathrm{LiH}^{5}$ The situation for $\mathrm{BeH}_{2}$ is very similar. There are in this case three domains, of which one corresponds to the core electron pair on $\mathrm{Be}$ and the other two are the $\mathrm{BeH}$ bond domains. As a consequence, there are in this case only three interdomain interaction terms. Two of them correspond to interactions between core domain of $\mathrm{Be}$ and bond domain of $\mathrm{BeH}$ bonds, and both are again small $(-0.065)$. What remains is the one bond-bond interdomain interaction term, and even if its value is slightly higher $(-0.070)$, the sum of the fluctuations over all domains must still be rather small so that the pair localization in the $\mathrm{BeH}$ bonds pair is also very high. The low value of the interbond interaction term can be in this case again attributed to the very high polarity of the $\mathrm{Be}-\mathrm{H}$ bond, which again, approaches the limit of nearly complete ionic bond. (AIM charge of the $\mathrm{H}$ atom in $\mathrm{BeH}_{2}$ is equal to 1.87 , which is quite comparable to the value 1.92 corresponding to the charge of $\mathrm{H}$ atom in $\mathrm{LiH}$.) We can thus see that the low fluctuation in the bond domains of $\mathrm{LiH}$ and $\mathrm{BeH}_{2}$ is, in fact, due to interference of two factors, namely high polarity of the corresponding bonds, and also the extremely simple structure of these molecules, which implies either the complete absence or only very limited number of interbond interaction terms that compensate the sum of individual domain fluctuations. In the case of more complex systems with less polar (covalent) bonds and with central atom participating in more bonds, the fluctuation in individual bond domains is necessarily reduced. This is the case of the molecule of $\mathrm{BH}_{4}^{(-)}, \mathrm{CH}_{4}$, and also of the above-discussed ethane molecule.

In the following part let us mention briefly the results of similar analysis for other simple systems $\left(\mathrm{C}_{2} \mathrm{H}_{4}, \mathrm{C}_{2} \mathrm{H}_{2}\right)$, for which the direct procedure used in the study ${ }^{27}$ would not also be applicable.

$$
\mathrm{C}_{2} \mathrm{H}_{4}
$$

The partitioning into ELF domains is in this case again quite straightforward, and clearly reflects the anticipated parallel with classical picture of bonding reflected in the structural formula. Consistent with this parallel, the ELF partitioning of this 16- 
electron molecule indeed yields eight domains populated by roughly two electrons. Two of them, populated roughly by 2.09 electrons, can straightforwardly be associated with the $1 \mathrm{~s}^{2}$ core electron pairs on each of the carbon atoms. In addition to these core pairs, which are again irrelevant for bonding, there is a group of four equivalent domains populated roughly by 2.08 electrons. These domains are classified as disynaptic $\mathrm{V}(\mathrm{CH})$ domains, and evidently each of them corresponds to one of the four $\mathrm{CH}$ bonds. The above interpretation is also straightforwardly corroborated by the comparison of the calculated fluctuation in these two types of domains ( 0.22 and 0.61 , respectively) with the analogous data for methane $e^{27}$ ( 0.239 and 0.622 , respectively). In addition to this, the close parallel of ELF and minimum fluctuation domains is also evident from practically identical values of the localization parameter $g(\Omega)$. After having demonstrated the close similarity between ELF and minimum fluctuation domains for the localization of core and $\mathrm{CH}$ bond electron pairs, it is thus necessary only to discuss the pair localization in two remaining domains, which evidently correspond to two electron pairs involved in the $\mathrm{CC}$ double bond. Each of these domains, which can be interpreted as banana components of the $\mathrm{CC}$ double bond, is populated roughly by 1.76 electrons. These values are again slightly lower than anticipated for the electron pair bonds, but in this case the deviation is not as dramatic and we can ignore it in the first approximation. (The situation where the population of certain ELF domains can significantly deviate from the anticipated electron pair is not exceptional and quite common is, for example, in the case of molecules with delocalized bonding. This, however, is not the case of the systems studied here where the parallel between ELF domain and localized electron pair chemical bond should be straightforward. The application of domain fluctuations to the description of delocalized bonding is discussed, for example, in the studies. ${ }^{36,37}$ ) Consistent with the slight deviation in the population of these domains are also the values of domain fluctuation (0.95) and localization index $g(\Omega)$, which both indicate the lowering of the pair localizability in these domains. This result is thus quite similar to what was observed above for the domain of the $\mathrm{CC}$ bond in ethane, and similar as in that case to the low value of the localization index cannot be interpreted in a sense of the failure of the electron pair model of chemical bonds. To support this claim it is again useful to look at the values of the interdomain interaction terms $F\left(\Omega_{\alpha}, \Omega_{\beta}\right)$. These values are summarized in Table 4 , and as it is possible to see, the values of the corresponding terms are again quite similar as in the case of ethane, and the only nonnegligible interactions exist just between electron pair bonds localized on the same carbon atom.

\section{$\mathrm{C}_{2} \mathrm{H}_{2}$}

As the final example, let us discuss in detail the results of our calculations for the molecule of ethine. In contrast to previous cases where the number of ELF domains always coincided with the number of localized electron pairs, the situation here is a bit more complex. The reason is that although the molecule contains 14 electrons, the ELF partitioning yields in this case only five domains. Their interpretation is, nevertheless, quite straightforward. Two of them, populated roughly by 2.09 electrons, correspond to the core electron pairs on the individual $\mathrm{C}$ atoms. Such an interpretation is also straightforwardly corroborated by the corresponding values of domain fluctuations and localization indices
(0.24 and 0.88 , respectively), which are straightforwardly comparable with the analogous data in other molecules. Simple and straightforward is also the interpretation of another two domains populated by 2.28 electrons. Although these values again in this case deviate from the ideal value of 2 , there are nevertheless good reasons to interpret them as disynaptic ${ }^{36} \mathrm{~V}(\mathrm{CH})$ domains corresponding to the $\mathrm{CH}$ bonds. This interpretation is also corroborated by the closeness of the values of domain fluctuations and localization indices to the analogous data for $\mathrm{CH}$ bonds in other molecules. What remains are three electron pairs of the $\mathrm{CC}$ triple bond. In contrast to the case of ethene where the individual electron pairs of the double $\mathrm{CC}$ bond could be separated into two different ELF domains, such a separation is not possible here, and the ELF partitioning associates the CC bonding with just a single domain. In the ideal case such a domain should thus be populated by six electrons, but because of slightly increased population of the domains corresponding to $\mathrm{CH}$ bonds, the population of this domain is a bit lower and attains roughly the value 5.27. Once again, we thus encounter the situation that the population of the domain deviates from the idealized anticipations. Although the deviation from the idealized limit 6 is in this case already quite considerable, there are again good reasons to assume that the association of individual domains with chemical bonds is in this case still reasonably plausible. As it will be shown, this is not always the case, and the examples of the apparent incompatibility of ELF domains with localized electron pair bonds will be discussed in detail in the following section. Before addressing these conflicting examples, however, let us pursue the analysis of the bonding arrangement in the molecule of the ethine. The quantity of the main interest will be the localization index of the domain corresponding to $\mathrm{CC}$ bond. In previous cases of ethane and the ethane molecule the localization of electron pairs in the domain of the $\mathrm{CC}$ bond was, namely, found to be even much lower than in the case of $\mathrm{CH}$ bond classified as questionable in the study. ${ }^{27}$ The situation here is in a sense, slightly easier. The localization index of the domain corresponding to the CC triple bond (0.75) is in this case, higher than the value 0.69 considered as a limit of "acceptable" pair localizability in the study. Although there is thus no need to question the applicability of the electron pair model of the chemical bond even using the criteria used in the study, ${ }^{27}$ it is nevertheless nice to see that the same conclusion is also corroborated by the detailed analysis of the interdomain interaction terms (Table 4). This result is very important because it demonstrates that the ELF partitioning of the molecule is often able to provide realistic alternative to the "best" partitioning anticipated by the loge theory, and that the electron pairs localized in these domains are remarkably reminiscent of classical chemical bonds of the Lewis model.

Unfortunately, such a straightforward correspondence is not observed in all cases, and the examples can be found where the above parallel is less convincing. In the following part we are going to discuss the results of our analysis for several simple molecules.

\section{$\mathrm{H}_{2} \mathrm{O}$ and $\mathrm{N}_{2}$}

Despite still being very simple, these molecules represent an interesting example of a systems for which the direct semiempirical procedure ${ }^{27}$ did not allow to find any single domain of valence 
density, which exhibits a minimum in its fluctuation, and instead, the motions of the valence electrons were found to be "so strongly intercorrelated." 27 In view of the above reported ability of ELF partitioning to provide a reasonably realistic model of bonding, it was of a special interest to subject these molecules to similar systematic scrutiny as in previous cases. Let us start first with the molecule of water. The ELF partitioning of this 10-electron molecule is still very straightforward and yields five domains. The first of them, populated roughly by 2.13 electrons, exhibits the fluctuation 0.33 , and the pair localization parameters is equal to 0.85 . These values clearly suggest that this monosynaptic domain should be associated with the core electron pair on oxygen. What about the remaining four domains? Intuitively, one would expect these domains to correspond to electron pairs of two $\mathrm{OH}$ bonds and two lone electron pairs on $\mathrm{O}$, and although such an interpretation can still be used for the purpose of the qualitative discussion, the more detailed insight into numerical characteristics of the corresponding domains shows that the parallel between these domains and the corresponding electron pairs is not in this case as convincing. The most important thing in this respect are the values of the population of the individual domains, whose deviations from the idealized expectations becomes more important. Thus, for example, the population of the disynaptic $\mathrm{V}(\mathrm{OH})$ domain associated with the $\mathrm{OH}$ bond is 1.69 , while the population of the monosynaptic $\mathrm{V}(\mathrm{O})$ domains associated with lone pairs are equal to 2.25 . These deviations then also reflected in the exalted values of electron fluctuation (0.77 and 1.04, respectively) and localization parameter $g(\Omega)(0.54$ and 0.54 , respectively). Although the situation that the population of individual domains can deviate from the idealized limits is quite normal and we have seen it also in many previous examples, the situation here is nevertheless a bit different. The problem is the increasing extent of these deviations, which makes it still more difficult to associate the domains with the localized electron pairs of chemical bonds. Although in the case of the water molecule a certain parallel can still be admitted, there are other, more "difficult" systems, for which such an association is more or less impossible. An example of such an extremely difficult system is the molecule of $\mathrm{N}_{2}$. This 14-electron molecule is isoelectronic to acetylene, and similar to what was observed there, the ELF partitioning yields only five domains. Two of them, populated roughly by 2.10 electrons, are again not very interesting because they evidently correspond to core electron pairs on $\mathrm{N}$ atoms. The remaining eight valence electrons thus necessarily must be distributed among the remaining three domains. On the basis of the analogy with acetylene it is possible to assume that two of these domains should be associated with lone electron pairs and the remaining one with the NN triple bond. This domain should thus in an ideal case again be populated by six electrons. Although already the example of acetylene demonstrated that one must be ready to accept that some deviations from the idealized populations are quite normal and do not necessarily imply the failure of the classical picture of the bonding in terms of electron pair bonds, the situation here is much more difficult, and the above intuitive interpretation of the domains seems to loose any real justification. Thus, for example, although the domain corresponding to NN triple bond is populated only by 3.55 electrons, the population of "lone pairs" is about 3.13. Consistent with these high deviations are also the values of domain fluctuations and localization param-
Table 5. Domain Populations, Fluctuations, and Pair Localization Parameters for the ELF Domains of Simple Molecules Where the Partitioning Is Not Compatible with the Electron Pair Model of the Chemical Bond.

\begin{tabular}{lllll}
\hline Molecule & Domain & $\overline{N(\Omega)}$ & $\Lambda(\Omega)$ & $g(\Omega)$ \\
\hline $\mathrm{H}_{2} \mathrm{O}$ & $\mathrm{C}(\mathrm{O})$ & 2.13 & 0.33 & 0.85 \\
& $\mathrm{~V}(\mathrm{OH})$ & 1.69 & 0.77 & 0.54 \\
& $\mathrm{~V}(\mathrm{O})$ & 2.25 & 1.04 & 0.54 \\
$\mathrm{~N}_{2}$ & $\mathrm{C}(\mathrm{N})$ & 2.10 & 0.27 & 0.87 \\
& $\mathrm{~V}(\mathrm{NN})$ & 3.55 & 1.47 & 0.59 \\
& $\mathrm{~V}(\mathrm{~N})$ & 3.13 & 1.10 & 0.65 \\
& & & & \\
\hline
\end{tabular}

eters (Table 5). We can thus see that the association of these domains with the classical concepts of chemical bond and/or lone pairs is practically unacceptable.

The reasons for this apparent failure of the ELF to yield the domains reminiscent of the "best" partitioning of the loge theory is not at this moment completely clear to us. Nevertheless, the fact that the decrease in the population of some domains is accompanied by nearly a complementary increase of the population of some other domains seems to suggest that the problem could be in the "incorrect" determination of the boundaries separating such domains in these conflicting cases. Although such an explanation is at this moment only speculative, we believe that the problem of explaining the possible failure of the ELF to determine in some cases the boundaries of the domains is worth pursuing. In this connection it is perhaps possible to mention that the same conclusion seems to be suggested by the preliminary results of our analysis of the Fermi holes averaged over ELF domains (R. Ponec, preliminary results, unpublished). If this is indeed the case, it seems reasonable to concentrate on the search of some other localization functions or procedures, in which the above problem of the "incorrect" determination of the boundaries of certain domains would be remedied. A possible progress in this respect could be, for example, the recent approach by Savin et al., ${ }^{31,38}$ who proposed to partition the molecule into the so-called maximum probability domains.

\section{Acknowledgment}

The authors thank Dr. E. Chamorro (Universidad Nacional Andres Bello, Chile) for the assistance with the modification of the TopMod program.

\section{References}

1. Lewis, G. N. J Am Chem Soc 1916, 38, 762.

2. Lennard-Jones, J. E. J Chem Phys 1952, 20, 2024.

3. Berlin, T. J Chem Phys 1951, 19, 208.

4. Ruedenberg, K. Rev Mod Phys 1962, 14, 326.

5. Daudel, R.; Bader, R. F. W.; Stephens, M. E.; Borrett, S. D. Can J Chem 1974, 52, 1310.

6. Levy, M. J Am Chem Soc 1976, 98, 6849. 
7. Salem, L. Nouv J Chim 1978, 2, 559.

8. Julg, A.; Julg, P. Int J Quantum Chem 1978, 13, 483.

9. Gopinathan, M. S.; Jug, K. Theor Chim Acta 1983, 63, 497, 511.

10. Cooper, D. L.; Gerratt, J.; Raimondi, M. Chem Rev 1991, 91, 929.

11. Ponec, R.; Strnad, M. Int J Quantum Chem 1994, 50, 43.

12. Savin, A.; Jepsen, O.; Flad, J.; Andersen, O. K.; Preuss, H.; von Schnering, H. G. Angew Chem Int Ed Engl 1992, 31, 187.

13. Coulson, C. Trans Faraday Soc 1942, 38, 433.

14. Lennard-Jones, J. E. Proc R Soc 1949, A198, 14.

15. Foster, J. M.; Boys, S. F. Rev Mod Phys 1960, 32, 300.

16. Edmiston, C.; Ruedenberg, K. J Chem Phys 1965, 43, 97.

17. Polák, R. Theor Chim Acta 1969, 14, 163.

18. Magnasco, V.; Perico, A. J Chem Phys 1972, 47, 738.

19. von Niessen, W. J Chem Phys 1972, 56, 4290.

20. Pipek, J.; Mezey, P. G. J Chem Phys 1989, 90, 4916.

21. Cioslowski, J. J Am Chem Soc 1991, 113, 4142.

22. Daudel, R.; Odiot, S.; Brion, H. J Chim Phys 1954, 51, 74.

23. Brion, H.; Daudel, R.; Odiot, S. J Chim Phys 1954, 51, 358.

24. Odiot, S.; Daudel, R.; Brion, H. J Chim Phys 1954, 51, 361.

25. Aslangul, C. C R Acad Sci Ser B 1971, 272, 1.

26. Bader, R. F. W.; Stephens, M. E. Chem Phys Lett 1974, 26, 445.

27. Bader, R. F. W.; Stephens, M. E. J Am Chem Soc 1975, 97, 7391

28. Ponec, R.; Uhlik, F. Theochem 1997, 391, 159.

29. Fradera, X.; Austen, M. A.; Bader, R. F. W. J Phys Chem A 1999, 103, 304.

30. Becke, D.; Edgecombe, K. E. J Chem Phys 1990, 92, 5397.
31. Gallegos, A.; Carbó-Dorca, R.; Lodier, F.; Cances, E.; Savin, A. J Comp Chem 2005, 26, 455.

32. Frisch, M. J.; Trucks, G. W.; Schlegel, H. B.; Scuseria, G. E.; Robb, M. A.; Cheeseman, J. R.; Zakrzewski, V. G.; Montgomery, J. A., Jr.; Stratmann, R. E.; Burant, J. C.; Dapprich, S.; Millam, J. M.; Daniels, A. D.; Kudin, K. N.; Strain, M. C.; Farkas, O.; Tomasi, J.; Barone, V.; Cossi, M.; Cammi, R.; Mennucci, B.; Pomelli, C.; Adamo, C.; Clifford, S.; Ochterski, J.; Petersson, G. A.; Ayala, P. Y.; Cui, Q.; Morokuma, K.; Malick, D. K.; Rabuck, A. D.; Raghavachari, K.; Foresman, J. B.; Cioslowski, J.; Ortiz, J. V.; Baboul, A. G.; Stefanov, B. B.; Liu, G.; Liashenko, A.; Piskorz, P.; Komaromi, I.; Gomperts, R.; Martin, R. L.; Fox, D. J.; Keith, T.; Al-Laham, M. A.; Peng, C. Y.; Nanayakkara, A.; Challacombe, M.; Gill, P. M. W.; Johnson, B.; Chen, W.; Wong, M. W.; Andres, J. L.; Gonzalez, C.; Head-Gordon, M.; Replogle, E. S.; Pople, J. A. Gaussian 98, Revision A.9; Gaussian, Inc.: Pittsburgh, PA, 1998.

33. Noury, S.; Krokidis, X.; Fuster, F.; Silvi, B. TopMod package, 1997.

34. Noury, S.; Colonna, F.; Savin, A.; Silvi, B. J Mol Struct 1998, 450, 59.

35. Chamorro, E.; Fuentealba, P.; Savin, A. J Comput Chem 2003, 24, 496.

36. Silvi, B. Phys Chem Chem Phys 2004, 6, 256.

37. Savin, A.; Silvi, B.; Colonna, F. Can J Chem 1996, 74, 1088.

38. Savin, A. In Reviews of Modern Quantum Chemistry (A Celebration of the Contributions of Robert G. Parr), K. D. Sen, Ed.; World Scientific, Singapore, 2002. 